\title{
Inter and intra band impurity-related absorption in (In,Ga)N/GaN QW under composition, size and impurity effects
}

\author{
Redouane En-nadir ${ }^{1}$, Haddou El Ghazi ${ }^{1,2, *}$, Anouar Jorio ${ }^{1}$, Izeddine Zorkani ${ }^{1}$ \\ ${ }^{1}$ LPS, FSDM, Sidi Mohammed Ben Abdellah University, Fes, Morocco \\ ${ }^{2}$ ENSAM Laboratory, ENSAM, Hassan II University, Casablanca, Morocco,
}

\begin{abstract}
In this paper, we theoretically investigate the impacts of Internal well composition, size and impurity's position on the inter valence-conduction bands and intra conduction band optical absorption in $\mathrm{GaN} /(\mathrm{In}, \mathrm{Ga}) \mathrm{N} / \mathrm{GaN}$ hetero-structure. Based on the numerically finite element method (FEM), the impurity's related Schrödinger equation is solved for the finite potential barrier considering the dielectric constant and effective-mass mismatches between the well and its surrounding matrix. Our results show that the absorption is strongly governed by the dipole matrix element and initial and final implied states transition energies. For a fixed barrier width, the absorption spectra are found red-shifted (blue-shifted) with increasing the well width (In-concentration). It is also shown that the impurity's absorption phenomenon is more pronounced for the off-center case compared to the on-center one. We conclude that the proper control of these parameters is required to best understanding of the optical absorption for solar cell applications.
\end{abstract}

\section{Introduction}

Since the discovery of low and wide energy band-gap materials, such as $\mathrm{InN}(0.78 \mathrm{eV})$ and $\mathrm{GaN}(3.42 \mathrm{eV})$ opened access to potential applications based on IIInitride semiconductors in the field of microelectronics and photonics. The combination of these two new materials gave a new InGaN material characterized by its adjustable band-gap energy which could be varies between $0.78 \mathrm{eV}$ and $3.42 \mathrm{eV}$, this property makes it possible to absorb the entire spectrum of visible light. This alloy has been several other advantages because of its direct band-gap, its operation in high temperature and high pressure devices, its thermal and chemical stability and especially its high absorption which makes it an excellent candidate for many industrial applications especially in optoelectronics domain, such as laser diodes (LDs), light-emitting diodes (LEDs), photo infrared detectors, laser amplifiers, optical memories and electro-optical high-speed modulator [1-8].

To improve optical and electrical properties of optoelectronic devices, researchers have been moved from the study of massive to Nano-structured materials, such as simple quantum wells (SQWs) [9].spherical quantum dot (SQDs) [10] quantum rings (QRs) [11] and quantum well wire(QWWs) [12]. Since Bastard's first calculations deal with the binding energy of the hydrogenic impurity in QW [13, 14], different low dimension structure (LDS) geometries such as cubical [15], rectangular [16], cylindrical [17], parabolic [18] and spherical QDs [19] have investigated. Study of linear and non-linear optical absorption coefficients $(\mathrm{OAC})$ in symmetric and asymmetric QW systems has attracted much interest both theoretically and experimentally. For instance, Peng et al. [20] have reported the Structure asymmetry effects in the optical gain of piezo-strained (In,Ga)N QW. Kostic and Stojanovic [21] reported the optical absorption of $\mathrm{Mg}$ doped layers and (In,Ga)N QW on c-plane and semipolar GaN structures. Yang et al. [22] have investigated the performance of $(\mathrm{In}, \mathrm{Ga}) \mathrm{N}$ based green laser diodes improved by using an asymmetric multi-quantum well active region. Additionally, Karimi et al. reported the second-order nonlinear optical properties in a strained $(\mathrm{In}, \mathrm{Ga}) \mathrm{N} /(\mathrm{Al}, \mathrm{Ga}) \mathrm{N}$ QW under the intense laser field excitations[23]. In the same trend, Saidi et al. reported the theoretical study of single and double resonant enhancement of second-harmonic generation in asymmetric (Al,Ga)N/GaN/(Al,Ga)N QW heterostructures [24]. Experimentally, many authors have investigated the interband and intraband transitions with and without impurity in the active layer. Sun et al. [25] have examined the interband transition in (Ga,NA)s/GaAs single QW by photoluminescence (PL) and absorption spectra. Sun et al. [26] reported a roomtemperature operating infrared photodetector based on the intersuband transition of (In, $\mathrm{As}) \mathrm{Sb} / \mathrm{GaSb} \mathrm{QW}$.

To the best of our knowledge, all theoretical studies cited above were based their calculations on the Ritzvariational approach with and without the impurity. In this work, we will concentrate to investigate the size, the impurity position and the indium concentration effects on the OACs related to intrasuband conduction and inter conduction-valence band transition in wurtzite unstrained $(\mathrm{In}, \mathrm{Ga}) \mathrm{N} / \mathrm{GaN}$ QW based the numerical finite elements method (FEM).

\footnotetext{
* Corresponding author: hadghazi@gmail.com
} 


\section{Theoretical framework}

In this paper, we considered unstrained wurtzite $\mathrm{GaN} /(\mathrm{In}, \mathrm{Ga}) \mathrm{N} / \mathrm{GaN}$ structure. In our model, the effective mass and dielectric constant mismatches are taken into consideration. In the framework of the effective mass and parabolic approximations, the Hamiltonian of an electron related to an off-center donor impurity in such structure is given by the following expression [27]:

$$
\mathrm{H}=-\frac{\square^{2}}{2} \vec{\nabla}\left(\frac{1}{\mathrm{~m}^{*}(\mathrm{e}, \mathrm{h})} \vec{\nabla}\right)+\mathrm{V}_{\mathrm{c}}(\mathrm{x})-\frac{\mathrm{e}^{2}}{\varepsilon_{0} \varepsilon^{*}\left|\overrightarrow{\mathrm{r}}-\overrightarrow{\mathrm{r}_{0}}\right|}
$$

Where, $\hbar$ is the Planck constant, $e$ is the electron charge, $\overrightarrow{r_{0}}$ is the impurity position, $\mathrm{m}^{*}(\mathrm{e}, \mathrm{h})$ is the electron and hole effective masses, $\quad\left|\overrightarrow{\mathrm{r}}-\overrightarrow{\mathrm{r}_{0}}\right|=$ $\sqrt{\left(\mathrm{x}-\mathrm{x}_{0}\right)^{2}+\left(\mathrm{y}-\mathrm{y}_{0}\right)^{2}+\left(\mathrm{z}-\mathrm{z}_{0}\right)^{2}}$ is the electron-impurity distance, $\varepsilon_{0}$ is the dielectric constant of vaccum and $\varepsilon_{r}^{*}$ is the main relative dielectric constant of the system. The indium-dependent growth direction confinement potential barrier, $V_{c}(x)$, is given as:

$V_{c}(x)=\left\{\begin{array}{lc}0 & L \leq x \leq L+l \\ Q\left[E_{g}^{G a N}-E_{g} \operatorname{In}_{x} G a_{1-x} N\right] & \text { elsewhere }\end{array}\right.$

Where, $Q(=0.7)$ is the conduction band-offset parameter, $E_{g}^{G a N}$ and $E_{g}^{I n N}$ are the band-gap energies of GaN and InN respectively which are the same as those used in Refs. [20]. The band-gap energy of $I n_{x} G a_{1-x} N$ ternary alloy as a function of the Indium-fraction is given by:

$\mathrm{E}_{\mathrm{g}}^{\mathrm{In}_{\mathrm{x}} \mathrm{Ga}_{1-\mathrm{x}} \mathrm{N}}=\mathrm{xE}_{\mathrm{g}}^{\mathrm{InN}}+(1-\mathrm{x}) \mathrm{E}_{\mathrm{g}}^{\mathrm{GaN}}-\mathrm{bx}(1-\mathrm{x}$

Where, $\mathrm{b}(=1.43 \mathrm{eV})$ is the bowing parameter for (In,Ga)N ternary alloy [28] Electron (hole) effective mass in $\operatorname{In}_{\mathrm{x}} \mathrm{Ga}_{1-\mathrm{x}} \mathrm{N}$ region is given by the following linear interpolation :

$m_{(e, \square)}^{* \operatorname{InGaN}}(x)=x m_{(e, \square)}^{* \operatorname{InN}}+(1-x) m_{(e, \square)}^{* G a N}$

The dielectric constant in InGaN is given by:

$\varepsilon_{\text {InGaN }}^{*}(\mathrm{x})=\mathrm{x} \varepsilon_{\mathrm{InN}}^{*}+(1-\mathrm{x}) \varepsilon_{\mathrm{GaN}}^{*}$

The relative refractive index $\left(n_{r}^{*}\right)$ in the well region is given by the following linear interpolation:

$n_{r}^{*}=x \sqrt{\varepsilon_{I n N}^{*}}+(1-x) \sqrt{\varepsilon_{G a N}^{*}}$

The eigenvalues and eigenvectors of the system are obtained by solving numerically the time-independent Schrödinger equation using the finite elements method. Linear and third-order nonlinear ACs are given as [29]:

$$
\begin{gathered}
\alpha^{1}(\omega)=\square \omega \sqrt{\frac{\mu}{\varepsilon_{r}^{*} \varepsilon_{0}}} \frac{\Gamma_{i f}\left|M_{f i}\right|^{2} \rho}{\left(E_{f i}-\square \omega\right)^{2}+\left(\square \Gamma_{i f}\right)^{2}} \\
-\sqrt{\frac{\mu}{\varepsilon_{\mathrm{r}}^{*}}}\left(\frac{\mathrm{I}}{2 \mathrm{n}_{\mathrm{r}}^{*} \varepsilon_{0} \mathrm{c}}\right) \frac{4 \Gamma_{\mathrm{if}}\left|\mathrm{M}_{\mathrm{fi}}\right|^{4} \rho \hbar \omega}{\left[\left(\mathrm{E}_{\mathrm{fi}}-\hbar \omega\right)^{2}+\left(\hbar \Gamma_{\mathrm{if}}\right)^{2}\right]^{2}}\left[1-\frac{\left|\mathrm{M}_{\mathrm{ff}}-\mathrm{M}_{\mathrm{ii}}\right|^{2}}{4\left|\mathrm{M}_{\mathrm{ff}}\right|^{2}} \frac{3 \mathrm{E}_{\mathrm{fi}}^{2}-4 \hbar \omega \mathrm{E}_{\mathrm{fi}}+(\hbar \omega)^{2}-\left(\hbar \Gamma_{\mathrm{if}}\right)^{2}}{\mathrm{E}_{\mathrm{fi}}^{2}-\left(\hbar \Gamma_{\mathrm{if}}\right)^{2}}\right]
\end{gathered}
$$

Therefore, the total OAC is defined as the sum of both contributions linear and third-order non-linear.

$\alpha^{\mathrm{T}}(\omega, \mathrm{I})=\alpha^{1}(\omega)+\alpha^{3}(\omega, \mathrm{I})$

Where, $I$ is the incident pump intensity, $n_{r}^{*}$ represents the refractive index of the semi-conductor and $c$ is the speed of light in vacuum. The electron density in the well is give as $\rho=N / V$ for which $N$ is the number of electrons in the well while $V$ is its volume. The incident photon angular frequency is $\omega$ while $\mu$ is the permeability of the system and $\tau_{f i}$ is the relaxation time between the implied states. The dipole transition matrix element between the lowest and the highest states is given by $M_{f i}=\left\langle\psi_{f}|e x| \psi_{i}\right\rangle$. The difference of the energy between final and initial states is given as $E_{f i}=E_{f}-E_{i}$. The dipole transitions between two deferent levels are allowed only when the selection rule $(\Delta l= \pm 1)$ is satisfied ( $l$ : the angular momentum quantum number). All necessary parameters used in this paper are the same as those used in Ref. [30].

\section{Results and discussion}

In this section, we will theoretically discuss behavior of impurity related OACs, energy difference between implied states and the probability of the presence of the electron in a symmetric SQW GaN/(In,Ga)N/GaN. Throughout the numerical calculations, we have used the effective units, i.e, the effective Bhor radius $\left(a_{b}^{*}=\right.$ $\left.\frac{\varepsilon_{0} \square^{2}}{m_{b}^{*} e^{2}}\right)$ as the unit of the width and the effective Rydberg energy $\left(R_{b}^{*}=\frac{m_{b}^{*} e^{4}}{2 \square^{2} \varepsilon_{0}^{2}}\right)$ as the unit of energy. $\mathrm{m}_{\mathrm{b}}^{*}$ is the effective mass in the barrier region ( $G a N)$. In order to obtain the necessary information to study the optical absorption in such structure, we have began to discuss the effects of the structure size, composition and impurity on the difference energies between the implied states.

Fig. 1 displays the energy difference between implied states as a function of the QW width for two different values of the Indium concentration, the barrier region width and the impurity's position for both transitions. Two cases are investigated concerning (1S-2P) for the intra-conduction subband transition and electron-hole recombination $\left(1 S_{e}-1 S_{h}\right)$ via the band gap for the interband transition. For all transitions, it is clear that with increasing the well size the difference energy presents two behaviors limiting by a critical value of the 
thickness structure independently of all parameters. This latter is around the effective Bohr radius.

One can observe from fig. 1 ( $a$ and $b$ ) that for fixed values of chemical structure and well size, $\Delta E$ is more affected for the thin well region corresponding to strong regime confinement. It is important to mention that with increasing the barrier size the difference energy increases too. For instance, the enhancement rate is about 5-10 meV for narrower well. It appears from fig.1 (b) that as the Indium concentration inside the well increases $\Delta E$ is dropped for a fixed value of the barrier size and for all well size. This can be explained by the fact that with increasing the Indium fraction the potential barrier augments which improves the confinement phenomena and then $\Delta \mathrm{E}$ can only decreases. Notice that for the interband recombination (fig.1: f), the drop is masked by the band gap energy. For this reason, $\Delta \mathrm{E}$ appears to be non sensitive to the well size. The same figure (c and e) shows that the displacement of the impurity has a great impact on the electronic states for all well size. It is shown that as the impurity is moved along the growth axis from the center $\Delta \mathrm{E}$ revealed an important improvement.
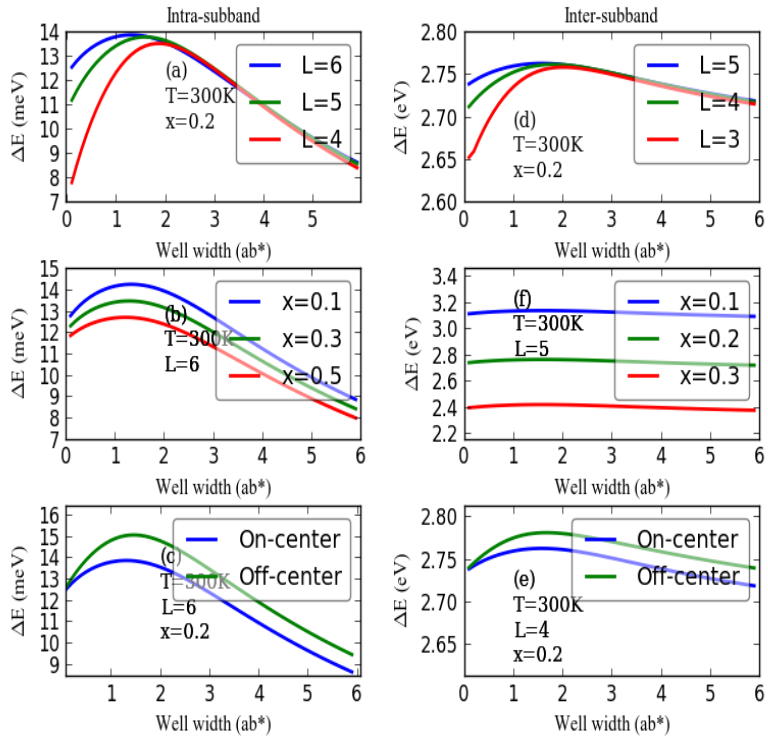

Figure 1: The difference energies between the implied states in GaN/(In, Ga)N/GaN QW versus the well width under the effects of the barrier width, the Inconcentration and the position of the impurity.

The dipole matrix element is one of the important parameter involved in the optical absorption phenomena and it's the key ingredient in estimation of gain or absorption. For this reason, $\left|M_{f i}\right|$ is plotted in fig. 2 for intra-conduction band according to the well width for different values of Indium concentration, barrier width and impurity's position. Notice that the same result is obtained for $\left|M_{f i}\right|$ corresponding to inter conductionvalence recombination. The main characteristic is that $\left|M_{f i}\right|$ increases versus the well width for all concentrations, barrier width and impurity positions. As the Indium fraction inside the ternary well increases,
$\left|M_{f i}\right|$ is found to be enhanced, whereas, it is dropped with increasing the barrier size. It is also shown that $\left|M_{f i}\right|$ is improved as the impurity is moved far away the structure center.

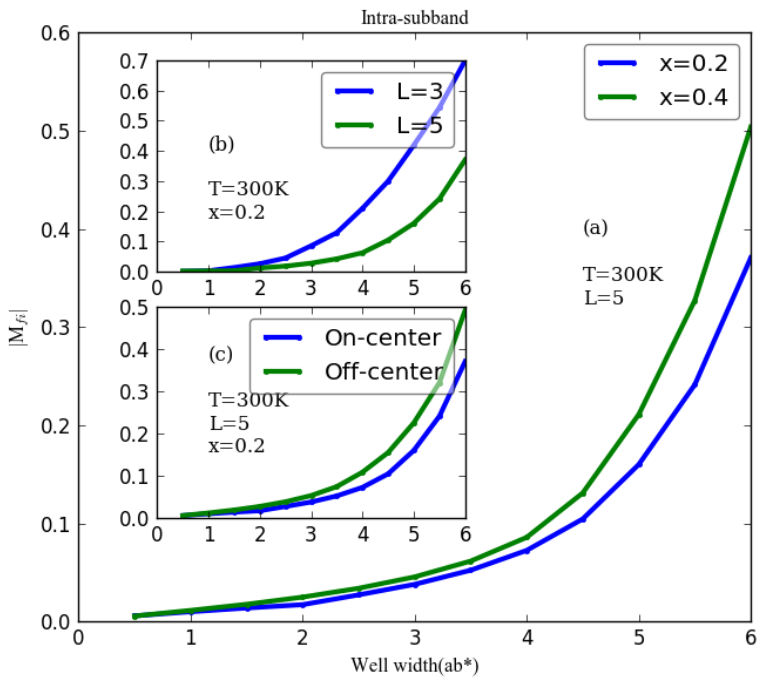

Fig. 2: The dipole matrix element according to the (GaN/(In,Ga)N/GaN QW width for two different values of the Indium concentrations, barrier's size and impurity's position.

The total optical absorption coefficients (OAC) related to two forms of transition, intrasuband and interband, are reported in Fig. 3 and Fig. 4 respectively according to the incident photon energy $(\hbar \omega)$. The effects of structure size, impurity position in and Infraction in the ternary have been investigated. The principle result is that the $\mathrm{OAC}$ is not monotonic and presents a maximum value for a critical incident photon energy governed by the above cited parameters.

It is shown that with increasing the well width $(l=$ $0.5,1$ and 1.5) a significant blue-shift is reported in Fig 3 (a) due to the increasing of the difference energy as discussed in Fig. 2. A considerable jump up of OAC amplitude is also observed. This can be explained by the competition of two parameters. $\left|M_{f i}\right|$ increases which enhancing the amplitude while $\Delta E$ increases also which induces a diminishing of the amplitude. These parameters act in the opposite sense but our results depict that impact of $\Delta E$ is masked by that of $\left|M_{f i}\right|$. We can also affirm that the third non-linear absorption coefficient is the responsible of this behavior.

The same figure (d) reveals also that the absorption phenomenon is more marked for the off-center impurity than for the on-center one. This can be assigned to the fact that as the impurity is moved far away the structure center the Coulomb interaction can only diminish and then the electron less attracted can be excited by the absorption of the incident photon more that the on-center one.

From Fig. 3 (c), it can be observed also that the increasing of the In-concentration inside the well induces a strong red-shift of the absorption spectra and a slight drop of their amplitude. These results are in perfect 
conformity with those presented above related to $\Delta E$ and $\left|M_{f i}\right|$. The same figure (b) reveals that the barrier width has also a great influence on the absorption spectra. It is obvious that the optical absorption spectra are redshifted according to the barrier width accompanied with a noticeable enhancement of their amplitude.
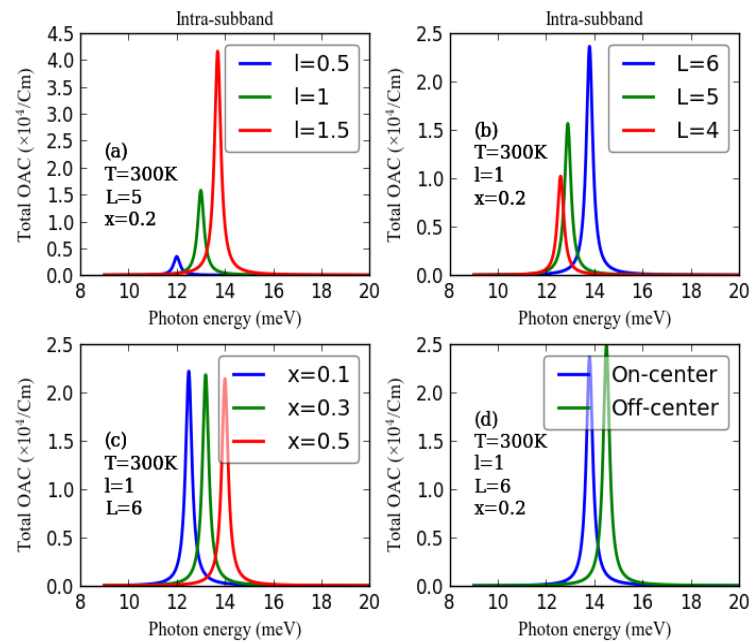

Fig. 3: Total optical absorption spectra of intra-subband transition as a function of the incident photon energy in $\mathrm{GaN} / \mathrm{InGaN} / \mathrm{GaN} \mathrm{QW}$ at room temperature. The effects of In-concentration, size and impurity are investigated.

Now, we turn to the inter valence-conduction bands recombination. For the first view, we clearly see that the effects of the well and the barrier sizes are less felt than in the case of intraband transition. The small change indicates that the effects of these parameters are shrouded by the others. On the one hand, the energy displacement around $10 \mathrm{meV}$ is masked by the band gap energy $(\mathrm{eV})$ which is the essential parameter implied in interband recombination. For this reason, the maximum of absorption spectra are located around $2.67 \mathrm{eV}$ for all structure sizes. On the other hand, the only effect is the increase in amplitude according to the well and barrier sizes.

It can be seen that the In-fraction in the ternary has a great influence on the absorption spectra. As can be seen from figure 4 (c), the absorption spectra are redshifted according to the In-composition. The physical reason is that when the In-fraction increases, the band gap energy dropped. For instance, this latter is $3.42 \mathrm{eV}$ and $2.67 \mathrm{eV}$ for $x=0$ and $x=0.2$ respectively. On the other hand, it is clearly seen that the amplitude diminishes as a function of the In-composition. It should be notice that as the In-composition is enhanced $\left|M_{f i}\right|$ increases and $\Delta E$ decreases which induces an amplitude enhancement but our results show a behavior contrary to that expected. The only explanation of this behavior can be assigned to the augmentation of the third nonlinear component of the absorption coefficient. Fig. 4 (d) reflects a weak dependence of the absorption coefficient on the impurity displacement. One can see that as the impurity is displaced far away the structure center the absorption spectra are slightly affected at the levels of the energy difference and the amplitude.
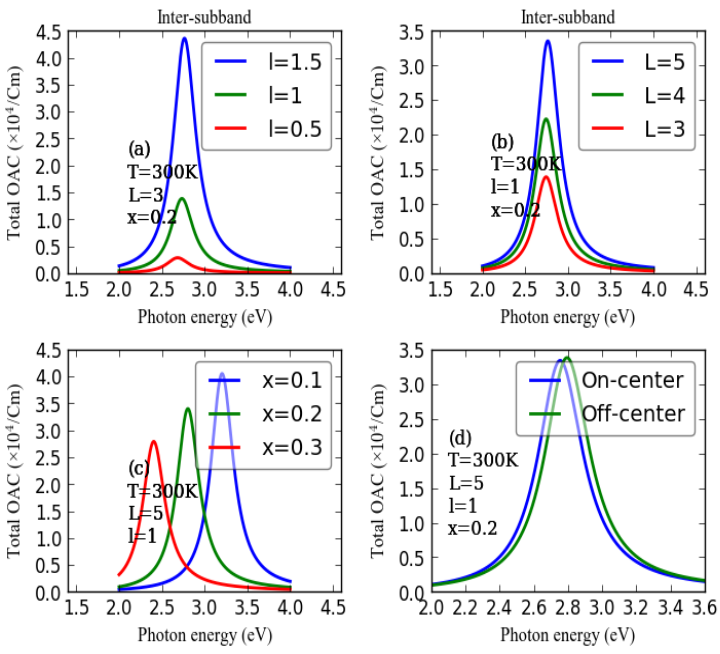

Fig. 4: Total optical absorption spectra of intersubband transition as a function of the incident photon energy of GaN/InGaN/GaN QW under the effects of In-concentration, size and impurity.

\section{Conclusion}

In summary, we have carried out a numerical simulation study of absorption coefficient based on the finite element method in unstrained $\mathrm{GaN} / \mathrm{InGaN} / \mathrm{GaN}$ QW. Two types of transitions of particular current interest are studied to exemplify the wide range of their applicability under well composition, impurity position and structure size. Our results show that these parameters can be adjusted to control the absorption coefficient for eventual solar cell application.

\section{References}

1. Y.S. Lee, Y.I. Jung, B.Y. Noh, I.K. Park, Appl. Phys. Express, 4, 112101 (2011)

2. K. Okamoto, Y. Kawakami, IEEE J. Selected Topics in Quantum Electronics, 15, 1199 (2009)

3. M. Tchernycheva, L. Nevou, L. Vivien, F. Julien, P. Kandaswamy, E. Monroy, A. Vardi, G. Bahir, P.S.S. (b), 247, 1622 (2010)

4. Y. Arakawa, IEEE Journal of Selected Topics in Quantum Electronics, 8, 823 (2002)

5. Y. Arakawa, T. Someya, K. Tachibana, IEICE Transactions on Electronics, 83, 564 (2000)

6. L. Ji, Y.-K. Su, S.-J. Chang, S. Tsai, S. Hung, R. Chuang, T. Fang, and T.-Y. Tsai, J. Vacuum Sci. \& Technology A: Vacuum, Surf. and Films, 22, 792-795 (2004)

7. H. El Ghazi, A. Jorio, I. Zorkani, Optics Communications, 331, 73-76 (2014)

8. Z. Romanowski, P. Kempisty, K. Sakowski, P. Stral’ 'gk, S. Krukowski, J. Phys. Chem. C, 114, 14 410-14 416 (2010) 
9. R. F. Nabiev, E. C. Vail, C. J. Chang-Hasnain, Laser Diodes and Applications, 2382 International Society for Optics and Photonics, 143-154, (1995)

10. K. Köksal, Y. Pavlyukh, Y. Pavlyukh, J. Berakdar, J. Beberakder, J. Sci. and Technology, 1, 4-6 (2011)

11. A. Fuhrer, S. Lüscher, T. Ihn, T. Heinzel, K. Ensslin, W. Wegscheider, M. Bichler, Nature, 413, 822 (2001)

12. M. Tsuchiya, J. Gaines, R. Yan, R. Simes, P. Holtz, L. Coldren, and P. Petroff, Phys. Rev. Let., 62, 466 (1989)

13. G. Bastard, Phys. Rev. B, 24, 4714 (1981)

14. J. Brum, G. Bastard, and C. Guillemot, Phys. Rev. B,30, 905 (1984)

15. F. Ribeiro and A. Latge, Phys. Rev. B, 50, 4913 (1994)

16. G. W. Bryant, Phys. Rev. B, 31, 7812 (1985)

17. N. P. Montenegro, J. Lopez-Gondar, L. Oliveira, Physical Review B, 43, 1824 (1991)

18. C. Bose, J. Appl. Phys., 83, 3089-3091 (1998)

19. N. Porras-Montenegro, S. Pérez-Merchancano, and A. Latge, J. Appli.Phys., 74, 7624-7626, (1993)

20. L.-H. Peng, Y.-C. Hsu, and C.-W. Chuang, IEEE J. of Selected Topics in Quantum electronics, 5, 756-764 (1999)

21. D. Sizov, R. Bhat, C.E. Zah, Journal of Applied Physics, 113, 203108 (2013)

22. J. Yang, D. Zhao, D. Jiang, X. Li, F. Liang, P. Chen, J. Zhu, Z. Liu, S. Liu, L. Zhang et al., Optics Express, 25, 9595-9602 (2017)

23. M. Karimi H. Vafaei, Superlattices and Microstructures, 78, 1-11 (2015)

24. I. Saidi, Journal of Applied Physics, 125, 185702 (2019)

25. B. Sun, D. Jiang, X. Luo, Z. Xu, Z. Pan, L. Li, and R. Wu, Appl. Phys. Let., 76, 2862 (2000)

26. L. Sun, L. Wang, J.-L. Lu, J. Liu, J. Fang, L.-L. Xie, Z.-B. Hao, H.-Q. Jia, W.-X. Wang, H. Chen, Chinese Physics B, 27, 047209 (2018)

27. H. El Ghazi, A. John Peter, Appl. Phys. A, 189, 125 (2019)

28. M. Anani, H. Abid, Z. Chama, C. Mathieu, A. Sayede, B. Khelifa, Microelectronics J., 38, 262-266 (2007)

29. S. Ortakaya, M. Kirak, and A. Guldeste, J. of Nonlinear Optical Phys. \& Mat., 26, 1750035 (2017)

30. H. El Ghazi, A. J. Peter, Physica B: Cond. Mat., 470-471, 164-68 (2015) 